\title{
Even-even gracefulness of some families of graphs
}

\author{
M.Sudha ${ }^{1}$, A. Chandra Babu ${ }^{2}$ \\ ${ }^{1}$ Assist. Professor, Department of Mathematics, Noorul Islam Centre for Higher Education, India. \\ ${ }^{2}$ Professor, Department of Mathematics, Noorul Islam Centre for Higher Education, India.
}

Abstract: In this paper, we prove that the Dumbbell graph, Star graph, Cartesian product $P_{2} \times C_{n}$ and $K_{1}+C_{n}$ are even-even graceful. The even-even graceful labeling of a graph $G$ with q edges means that there is an injection $f: E(G) \rightarrow\{2,4, \ldots, 2 q\}$ so that induced map $f^{*}: V(G) \rightarrow\{0,2, \ldots,(2 k-2)\}$ defined by $f^{*}(x) \equiv \Sigma f(x, y)(\bmod 2 k)$ where $k=\max \{p, q\}$ makes all distinct and even.

Keywords: Even-even graceful labeling, Dumbbell graph, Star graph and wheel graph.

\section{Introduction}

Most graph labeling methods trace their origin to one introduced by Rosa [1] in 1967, or the one given by Graham and Sloane [2] in 1980. Rosa [1] called a function $\mathrm{f}$ a $\beta$-valuation of a graph $\mathrm{G}$ with q edges if $\mathrm{f}$ is an injection from the vertices of $G$ to the set $\{0,1, \ldots, q\}$ such that, when each edge $x y$ is assigned the label $\mid f(x)$ $-\mathrm{f}(\mathrm{y}) \mid$, the resulting edge labels are distinct. Golomb subsequently called such labeling graceful and this is now the popular term. For all terminology and notation Bondy[3] has been followed. Solairaju and Chithra [4] have introduced the concept of edge-odd gracefulness. Gayathri and Duraisamy have introduced the concept of even edge-graceful labeling. A graph is even vertex-graceful if there exists an injective map $\mathrm{f}: \mathrm{E}(\mathrm{G}) \rightarrow\{1,2, \ldots, 2 \mathrm{q}\}$ so that the induced map $\mathrm{f}^{+}: \mathrm{V}(\mathrm{G}) \rightarrow\{0,2,4, \ldots, 2 \mathrm{k}-2\}$ defined by $\mathrm{f}^{+}(\mathrm{x})=\Sigma \mathrm{f}(\mathrm{xy})(\bmod 2 \mathrm{k})$ where $\mathrm{k}=\max \{\mathrm{p}, \mathrm{q}\}$ makes all distinct. R.Sridevi, S. Navaneethakrishnan, A.Nagarajan and K. Nagarajan [5] have introduced the concept of odd-even gracefulness. They proved that some well known graphs namely $P_{n}, P^{+}{ }_{\mathrm{n}}, K_{1, n}, K_{1,2, n}, K_{m, n}$, $B_{m, n}$ are odd-even graceful. In this paper we introduce the definition even-even gracefulness and also prove that some well known graphs namely $S_{n}, D(m, n)$ and $P_{2} \times C_{n}$ etc are even-even graceful.

\section{Definition1.1}

The odd-even graceful labeling of a graph $\mathrm{G}$ with $\mathrm{q}$ edges is an injection $\mathrm{f}: \mathrm{V}(\mathrm{G}) \rightarrow\{1,3,5, \ldots, 2 \mathrm{q}+1\}$ such that, when each edge uv is assigned the label $|\mathrm{f}(\mathrm{u})-\mathrm{f}(\mathrm{v})|$, the resulting edge labels are $\{2,4,6, \ldots, 2 \mathrm{q}\}$. A graph which admits an odd-even graceful labeling is called an odd-even graceful graph.

\section{Definition1.2}

A graph is even vertex graceful if there exists an injective map $f: E(G) \rightarrow\{1,2, \ldots, 2 q\}$ so that the induced map $\mathrm{f}^{+}: \mathrm{V}(\mathrm{G}) \rightarrow\{0,2,4, \ldots, 2 \mathrm{k}-2\}$ defined by $\mathrm{f}^{+}(\mathrm{x})=\mathrm{f}(\mathrm{xy})(\bmod 2 \mathrm{k})$ where $\mathrm{k}=\max \{\mathrm{p}, \mathrm{q}\}$ makes all distinct.

\section{Definition1.3}

A graph is even-even graceful if there exists an injective map $\mathrm{f:} E(\mathrm{G}) \rightarrow\{2,4, \ldots, 2 \mathrm{q}\}$ so that the induced map $\mathrm{f}^{*}: \mathrm{V}(\mathrm{G}) \rightarrow\{0,2, \ldots,(2 \mathrm{k}-2)\}$ defined by $\mathrm{f}^{*}(\mathrm{x}) \equiv \Sigma \mathrm{f}(\mathrm{x}, \mathrm{y})(\bmod 2 \mathrm{k})$ where $\mathrm{k}=\max \{\mathrm{p}, \mathrm{q}\}$ makes all distinct and even.

\section{Main Results}

Definition 2.1 A star $S_{n}$ is the complete bipartite graph $K_{1, n}$. It is a tree with one internal node and $n$ leaves.

Theorem 2.1 A star graph $S_{n}$ is even-even graceful when $n$ is even.

Proof: Let $\mathrm{G}$ be a star graph with $\mathrm{n}+1$ vertices and $\mathrm{n}$ edges.

Let $\left\{\mathrm{e}_{1}, \mathrm{e}_{2}, \ldots, \mathrm{e}_{\mathrm{n}}\right\}$ be the edge set of $\mathrm{S}_{\mathrm{n}}$.

Define $\mathrm{f}: \mathrm{E}(\mathrm{G}) \rightarrow\{2,4, \ldots, 2 q\}$ such that $($ here $\mathrm{q}=\mathrm{n}) \mathrm{f}\left(\mathrm{e}_{\mathrm{i}}\right)=2 \mathrm{i} ; \mathrm{i}=1,2, \ldots, \mathrm{n}$.

The internal vertex of $S_{n}$ has induced label

$$
\begin{aligned}
2+4+6+\ldots+2 \mathrm{n} & =2(1+2+3+\ldots+\mathrm{n}) \\
& =\frac{2 n(n+1)}{2} \\
& =\mathrm{n}(\mathrm{n}+1) \\
& =\mathrm{n} \cdot \mathrm{k} \text { where } \mathrm{k}=\mathrm{p}=\mathrm{n}+1
\end{aligned}
$$


$2+4+6+\ldots+2 \mathrm{n} \equiv 0(\bmod 2 \mathrm{k})$ when $\mathrm{n}$ is even

Hence, the induced label of internal vertex is ' 0 ' and other vertices have induced label from 2 to $2 \mathrm{n}$.

Example 2.1 The star graph $\mathrm{S}_{4}$ is even-even graceful.

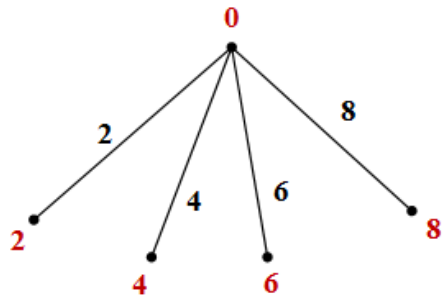

Figure: 1

Definition 2.2 The Dumbbell graph $D(m, n)$ is formed by two disconnected cycles $C_{m}$ and $C_{n}$ joined by an edge. Theorem 2.2 Dumbbell graph $D(m, n)$ is even-even graceful for $m=n$.

Proof: For any $n \geq 3$, the Dumbbell graph $D(m, n)$ has $2 n$ vertices and $2 n+1$ edges. Let $\left\{e_{1}, e_{2}, \ldots, e_{n}\right\}$ be the edge set of the first cycle $C_{n}$. 'e $\mathrm{e}_{\mathrm{n}+1}$ ' be a connecting edge. $\left\{\mathrm{e}_{\mathrm{n}+2}, \mathrm{e}_{\mathrm{n}+3}, \ldots, \mathrm{e}_{2 \mathrm{n}+1}\right\}$ be the edge set of the second cycle $C_{n}$.

We begin with the first cycle $C_{n}$ by labeling 2 to 2 n to each edge anticlockwise consecutively from one side of the connected vertex. Then we label $2 n+2$ to the connected edge .Finally we label $2 n+4$ to $4 n+2$ to each edge of the second cycle $C_{n}$ clockwise from one side of the connecting vertex.

Hence, the vertices of first cycle $C_{n}$ has induced labels $\mathrm{f}\left(\mathrm{v}_{\mathrm{i}}\right)=4 \mathrm{i}-2 ; \mathrm{i}=1,2, \ldots, \mathrm{n}$ and the vertices of second cycle $C_{n}$ has induced labels $\mathrm{f}\left(\mathrm{v}_{\mathrm{i}}{ }^{1}\right)=4 \mathrm{i} ; \mathrm{i}=1,2,3, \ldots, \mathrm{n}$.

Example: 2.2 The Dumbbell graph with even-even graceful labeling.

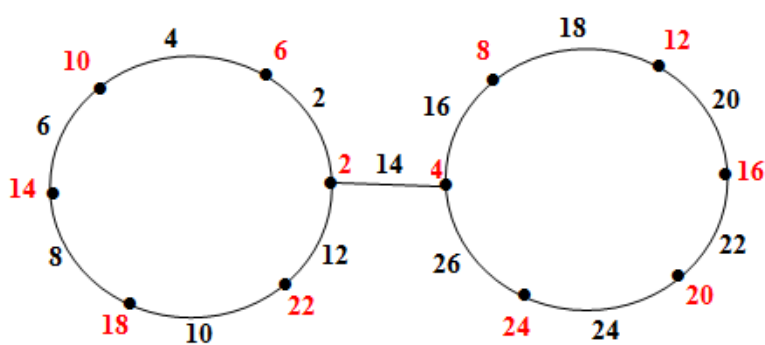

Figure: 2

Theorem 2.3 The ladder graph $P_{2} \times C_{n}$ is even-even graceful.

Proof:

The graph $P_{2} \times C_{n}$ has 2 v vertices and 3 n edges. First we consider $\mathrm{e}_{1}$ and $\mathrm{e}_{3 \mathrm{n}}$, the two outer edges of $P_{2} \times C_{n}$. Let $\left\{\mathrm{e}_{2}, \mathrm{e}_{3}, \ldots, \mathrm{e}_{\mathrm{n}}\right\}$ be the edge set of one of the long sides of the ladder and $\left\{\mathrm{e}_{2 n+1}, \mathrm{e}_{2 n+2}, \ldots, \mathrm{e}_{3 \mathrm{n}-1}\right\}$ be the edge set of the other long side of ladder. Finally let $\left\{\mathrm{e}_{\mathrm{n}+1}, \mathrm{e}_{\mathrm{n}+2}, \ldots, \mathrm{e}_{2 \mathrm{n}}\right\}$ be the edge set of rungs of ladder.

Define $\mathrm{f}: \mathrm{E}(\mathrm{G}) \rightarrow\{2,4, \ldots, 2 q\}$ such that

$\mathrm{f}\left(\mathrm{e}_{1}\right)=2 ; \mathrm{f}\left(\mathrm{e}_{3 \mathrm{n}}\right)=6 \mathrm{n}$ and $\mathrm{f}\left(\mathrm{e}_{\mathrm{i}}\right)=2 \mathrm{i} ; \quad \mathrm{i}=2,3, \ldots, 3 \mathrm{n}-1$.

From the above labeling, the induced vertex labels of the two paths $P_{n}$ are

$\mathrm{f}^{+}\left(\mathrm{v}_{\mathrm{i}}\right)=4(\mathrm{n}+1)+2 \mathrm{i}$ for $\mathrm{i}=1,2, \ldots, \mathrm{n}-3$;

$\mathrm{f}^{+}\left(\mathrm{v}_{\mathrm{n}-2}\right)=0 ; \mathrm{f}^{+}\left(\mathrm{v}_{\mathrm{n}-1}\right)=2 \& \mathrm{f}^{+}\left(\mathrm{v}_{\mathrm{n}}\right)=4(\mathrm{n}+1)$;

$\mathrm{f}^{+}\left(\mathrm{v}_{1}^{1}\right)=2(\mathrm{n}+1)$;

$\mathrm{f}^{+}\left(\mathrm{v}_{\mathrm{i}}^{1}\right)=2 \mathrm{i}$ for $\mathrm{i}=2,3, \ldots, \mathrm{n}$.

Hence the graph $P_{2} \times C_{n}$ is an even-even graceful. 


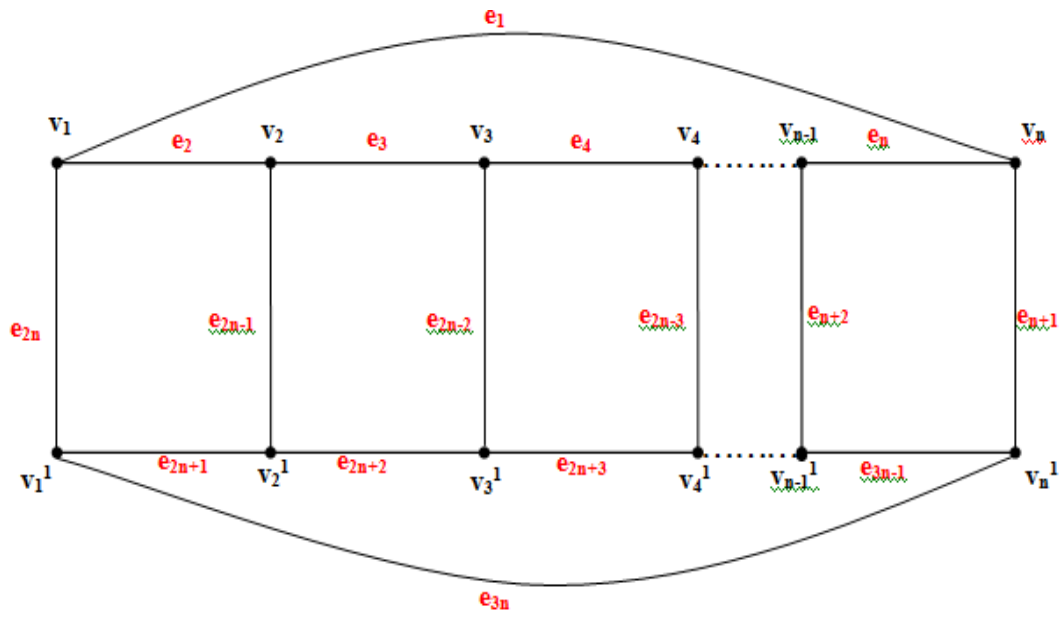

Figure: 3

Example: 2.3 The following figure shows that the graph $P_{2} \times C_{5}$ is even-even graceful.

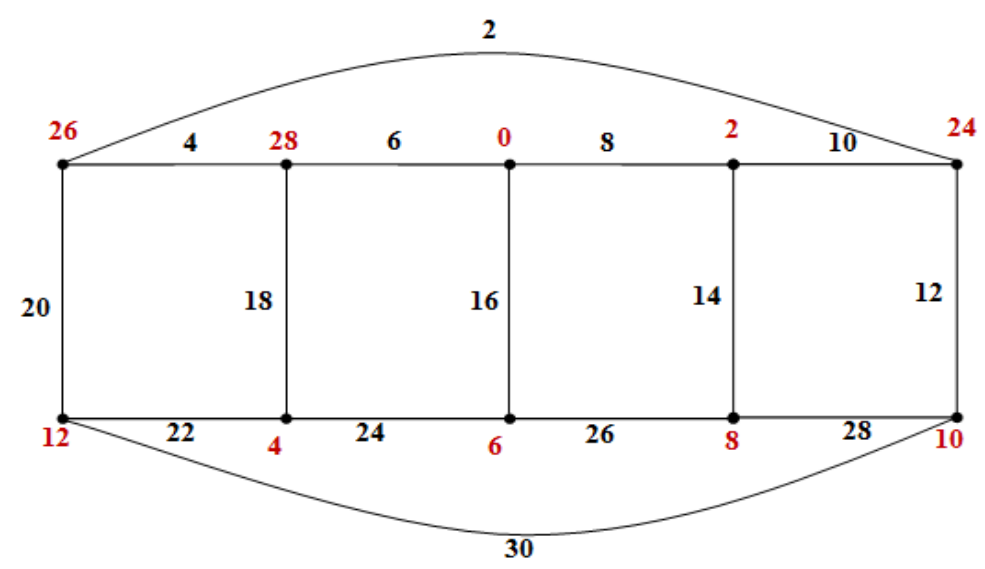

Figure: 4

\section{Definition 2.3}

The wheel, $\mathrm{W}_{\mathrm{n}}$, is the graph obtained by joining every vertex of the cycle $\mathrm{C}_{\mathrm{n}}$ to exactly one isolated vertex called the center. The edges incident to the center are called spokes.

Theorem 2.4 The wheel $\mathrm{W}_{\mathrm{n}}$ is even-even graceful when $\mathrm{n} \equiv 0(\bmod 4)$

Proof:

The graph $\mathrm{W}_{\mathrm{n}}$ has $\mathrm{n}+1$ vertices and $2 \mathrm{n}$ edges. Let $\left\{\mathrm{e}_{1}, \mathrm{e}_{2}, \mathrm{e}_{3}, \ldots, \mathrm{e}_{\mathrm{n}}\right\}$ be the edge set of the spokes and $\left\{\mathrm{e}_{\mathrm{n}+1}, \mathrm{e}_{\mathrm{n}+2}, \ldots, \mathrm{e}_{2 \mathrm{n}}\right\}$ be the edge set of consecutive cycle. Let ' $\mathrm{v}_{0}$ 'be a center vertex and $\mathrm{v}_{1}, \mathrm{v}_{2}, \ldots, \mathrm{v}_{\mathrm{n}}$ be the consecutive cycle vertices.

Define $\mathrm{f}: \mathrm{E}(\mathrm{G}) \rightarrow\{2,4, \ldots, 2 q\}$ such that

$\mathrm{f}\left(\mathrm{v}_{0} \mathrm{v}_{\mathrm{i}}\right)=2 \mathrm{i}$ for $\mathrm{i}=1,2, \ldots . \mathrm{n}$

$\mathrm{f}\left(\mathrm{v}_{\mathrm{i}} \mathrm{v}_{\mathrm{n}}\right)=4 \mathrm{n}-2(\mathrm{i}-1)$ for $\mathrm{i}=1,2, \ldots, \mathrm{n}$

Hence the induced mapping are $\mathrm{f}^{*}\left(\mathrm{v}_{0}\right)=\mathrm{n}$;

$$
\begin{aligned}
& \mathrm{f}^{*}\left(\mathrm{v}_{1}\right)=2 \mathrm{n}+4 ; \\
& \mathrm{f}^{*}\left(\mathrm{v}_{2}\right)=2 ; \\
& \mathrm{f}^{*}\left(\mathrm{v}_{3}\right)=0 \text { and } \\
& \mathrm{f}^{*}\left(\mathrm{v}_{\mathrm{i}}\right)=4 \mathrm{n}-2 \mathrm{i}+6 \text { for } \mathrm{i}=4,5, \ldots, \mathrm{n} .
\end{aligned}
$$




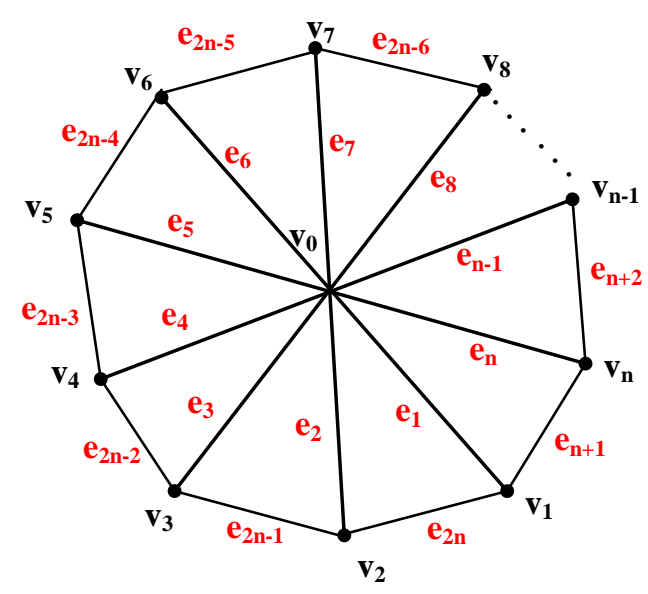

Figure: 5

Example: 2.4 The following figure shows that the graph $\mathrm{W}_{8}$ is an even-even graceful.

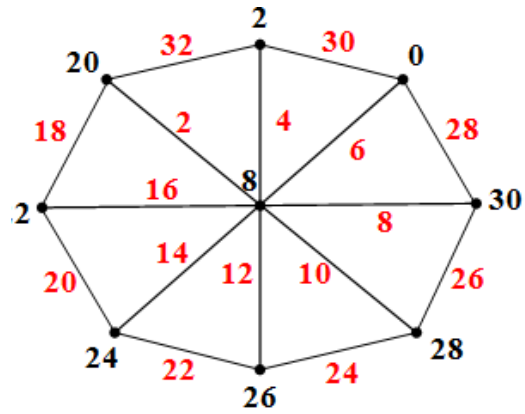

Figure: 6

Definition 2.4 The join of graphs $K_{1}$ and $C_{n}, K_{1}+C_{n}$, is obtained by joining every vertex of $K_{1}$ with every vertex of $\mathrm{C}_{\mathrm{n}}$ with an edge.

Theorem 2.5 The graph $K_{1}+C_{n}$ is eve-even graceful if $n$ is a multiple of 4 .

Proof: The graph $K_{1}+C_{n}$ has $n+1$ vertices and $2 n$ edges. Let ' $v$ ' be vertex of $K_{1}$ and $v_{1}, v_{2}, \ldots, v_{n}$ be a vertices of the cycle. Start at the first edge which are incident to the $\mathrm{K}_{1}$ with 2 and continue in strictly increasing order by 2. $\therefore$ The smallest edge label is 2 and largest edge label is $2 \mathrm{n}$.

Similarly, label the edges of $\mathrm{C}_{n}$, start from right hand side with $2 \mathrm{n}+2$ and continue in strictly increasing order by 2. So the smallest edge label of $C_{n}$ is $2 n+2$ and largest edge label is $4 n$.

Hence the induced labels of vertices are,

$f^{*}(v)=n ; f^{*}\left(v_{1}\right)=0 ; f^{*}\left(v_{n}\right)=2$ and $f^{*}\left(v_{i}\right)=4 n-2 i+2$ if $n=2,4, \ldots, n-1$ 


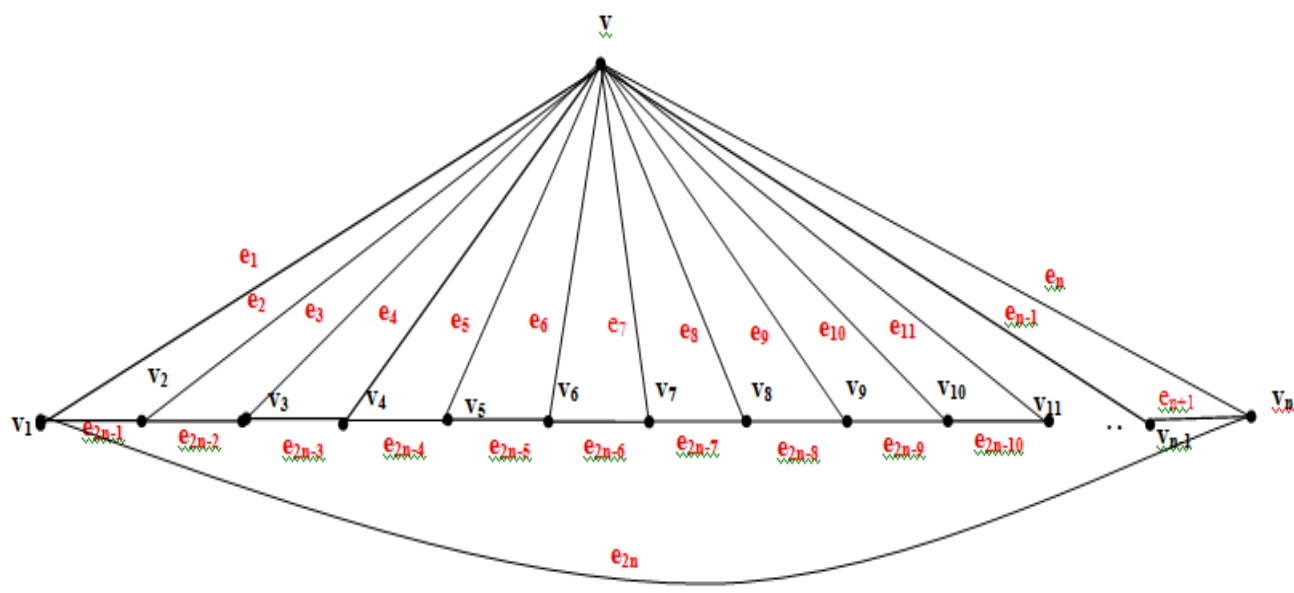

Figure: 7

Example: 2.5 The graph $\mathrm{K}_{1}+\mathrm{C}_{12}$ and its even-even graceful labeling are shown in the following Figure.

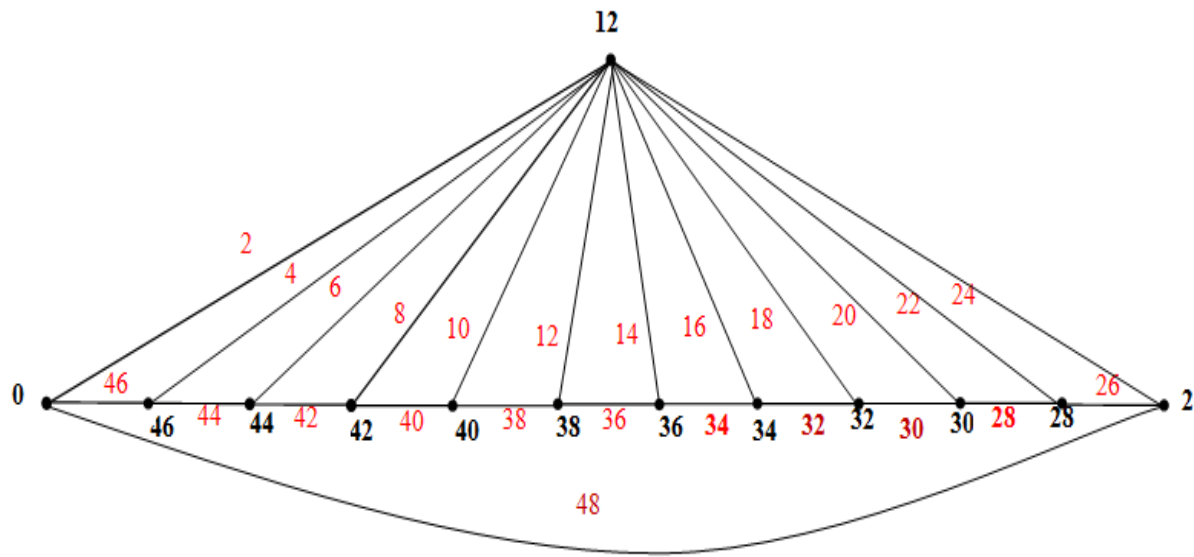

Figure: 8

\section{Conclusion}

In this paper we have introduced the definition for 'even-even graceful labeling'. We have proved that the Dumbbell graph, Star graph, Cartesian product $P_{2} \times C_{n}$ and $\mathrm{K}_{1}+\mathrm{C}_{\mathrm{n}}$ are all even-even graceful. We have also proved that the wheel $\mathrm{W}_{\mathrm{n}}$ is even-even graceful when $\mathrm{n} \equiv 0(\bmod 4)$.

\section{References}

[1]. A.Rosa, "On certain valuations of the vertices of a graph", Theory of Graphs (Internat. Symposium, Rome, July 1966), Gordon and Breach, N. Y. and Dunod Paris (1967) 349-355.

[2]. R. L. Graham and N. J. A. Sloane, "On additive bases and harmonious graphs", SIAM

[3]. J. Alg. Discrete Math., 1 (1980) 382-404. [1].

[4]. J. Bondy and U. Murty, Graph Theory with Applications, North- Holland, New York (1979).Christian Barrientos, "Odd-Graceful Labelings of Trees of Diameter 5", AKCE J.Graphs. Combin., 6, No. 2 (2009) .

[5]. A. Solairaju and K. Chithra, Edge-odd graceful labeling of some graphs, Proceedings of the ICMCS, 1 (2008) 101-107.

[6]. R. Sridevi, S. Navaneethakrishnan, A. Nagarajan and K. Nagarajan,” Odd-Even graceful graphs”, J. Appl. Math. \& Informatics Vol. 30(2012), No. 5 - 6, pp. $913-923$.

[7]. J.A.Gallian, A dynamic survey of graph labeling, Electronic, J.Comb,

[8]. Sin-Min Lee,Kuo-Jue Chen,Yung-Chin Wang "On the Edge graceful spectra of cycles with one chord and dumbbell graphs", Congressus Numerantium 170 (2004), pp. 171-183. 